\title{
LA ENSEÑANZA DE LA FÍSICA EXPERIMENTAL EN LA EUROPA DEL SIGLO XVIII
}

\author{
Víctor GUIJARRO MORA \\ Universidad Rey Juan Carlos
}

Introducción

La física experimental se consolidó a lo largo del siglo XVIII como una disciplina que incorporaba los hallazgos y espectaculares logros del primer período de la Revolución Científica en el ámbito de las ciencias físicas ${ }^{1}$. Era así mismo un instrumento empleado por los "modernos" en contra de las, según ellos, anticuadas teorías basadas en el aristotelismo, o lo que venía a ser lo mismo, en contra del espíritu de sistema y del estudio de la naturaleza guiado por la disputa verbal. El adjetivo "experimental" que acompañaban a su estilo de hacer física significaba una defensa de la superioridad de su método, basado en la observación y la comprobación de las hipótesis, sobre las improductivas prácticas argumentativas de los antiguos.

Así, la física experimental se articuló como un estilo de investigar los procesos naturales caracterizado por la adopción de diferentes estrategias, entre las que destacan: la búsqueda de nuevas propiedades y sus relaciones; la realización

1 Sobre la física de este siglo, I. B. CoHEN, Franklin and Newton. An Inquiry into Speculative Newtonian Experimental Science and Franklin's Work in Electricity as an Example thereof, Philadelphia: The Philosophical Society, 1956; J. L. HeILBRON, Electricity in the 17th and 18th Centuries. A study of early modern physics, Berkeley: University of California Press, 1979; H. GUERLAC, trabajos reunidos en Newton on the Continent, Ithaca: Cornell University Press, 1981 y en Essays and Papers in the History of Modern Science, Baltimore: The John Hopkins University Press, 1977 y Thomas L. Hankins, Jean D'Alembert. Science and the Enlightenment, Oxford: Clarendon Press, 1970. 
de mediciones asociadas con propiedades físicas (destinadas a la obtención de leyes simples); el mejoramiento de las técnicas de laboratorio y del utillaje empleado y el rechazo de las matemáticas (o, al menos, de la geometría) como vía de comprensión de la naturaleza. Se aplicaban preferentemente en aquellos campos que contaban con una escasa tradición, como era el caso del calor, la electricidad, el magnetismo y las propiedades del aire. De esta forma se continuaban poniendo en práctica las aspiraciones y métodos empleados en el siglo anterior por Robert Boyle.

Pero este campo de estudios además adquirió una dimensión más amplia, centrada esta vez en la promoción y defensa de las novedades y firmeza de los últimos resultados científicos, donde destacaban especialmente las aportaciones de Isaac Newton. Difundir la física experimental significaba, al mismo tiempo, de acuerdo con sus promotores, asumir un camino seguro de hacer ciencia que se había iniciado con las aportaciones de, por citar algunos ejemplos, Boyle, G. Galilei, R. Hooke, B. Pascal, E. Torricelli y C. Huygens y había culminado con el autor de los Principia y de la $O_{\text {ptica. }}$. Así pues, aquellos países, como Inglaterra, cuyos filósofos naturales se declararon newtonianos, muy pronto introdujeron en sus aulas las enseñanzas y manuales asociados con la física experimental.

En este texto, por tanto, se verá el nivel de difusión que conocieron en Europa estos estudios, los métodos empleados en su enseñanza, sus contenidos y el proceso de incorporación en España, donde destaca el caso de los Reales Estudios de San Isidro.

\section{Institucionalización de la física experimental}

La física dominante en los centros de educación superior estaba articulada desde el siglo XIII en torno al corpus aristotélico ${ }^{2}$. Se impartía en la Facultad de Artes, que era considerada una facultad menor o preparatoria para las otras facultades superiores, es decir, para la de Medicina, Leyes o Cánones. El curso comprendía una physica generalis, donde se trataba de la forma, la sustancia, la

2 Sobre los saberes cientificos en la universidad, P. KIBRE y N. C. Siriasi, "The Institutional Setting: The Universities", en D. C. LINDBERG (Ed.), Science in the Middle Ages. Chicago: University of Chicago Press, 1978, págs. 120-144. 
causa, el accidente, el lugar, el tiempo, la necesidad y el movimiento. Estas categorías se aplicaban posteriormente a la explicación de los fenómenos del mundo orgánico e inorgánico, estudiados en el apartado de la physica particularis, el cual estaba constituido por las siguientes partes: De caelo, De generatione et corruptione, Meteorologica, De partibus animalium y De anima. De esta forma se organizaba el curriculum del Curso Filosófico, que se mantuvo vigente hasta el siglo XVII, en algunos casos, $y$ hasta bien entrado el siglo XVIII, en otros muchos. Entendida de esta manera, la física agrupaba todos los estudios referentes a la naturaleza, desde el mundo sublunar al supralunar, pasando por la clasificación de los animales, plantas y minerales y terminando con el examen del hombre. Por tanto, nos encontramos con una disciplina muy distinta de lo que actualmente entendemos por física. Por otra parte, y esto representa una diferencia más, muy difícilmente admitía la experimentación, y mucho menos el uso de las matemáticas.

Solamente en la segunda mitad del siglo XVII comenzaron a introducirse demostraciones experimentales en algunos colegios de jesuitas (especialmente en los franceses) ${ }^{3}$ y universidades alemanas. Junto a estos establecimientos, los que sí mantenían en cualquier caso una reconocida tradición en el uso de este tipo de demostraciones eran las academias científicas, situación en la que se encontraban la Accademia del Cimento (fundada en Florencia en el año 1657), la Royal Society de Londres (1660) y la Académie Royale des Sciences de París (1666).

A pesar de estos cambios, el paso realmente fundamental para la incorporación de la física experimental en las universidades se produce en el año 1700 . Como ya hemos afirmado este saber significaba algo más que la utilización del experimento; era, o eso se pretendía que fuera, la nueva física. Por tanto, exigía unas transformaciones más radicales, cambios, producidos a partir de la fecha indicada en las universidades inglesas, que consistieron fundamentalmente en la restricción de sus contenidos ${ }^{4}$ y en su organización de una forma interrelacionada. En concreto, es en la universidad de Oxford donde se acoge por primera vez un curso de física experimental en el sentido que lo estamos empleando aquí. Se trata de las lecciones impartidas desde 1694 por J. Keill, cuyo

3 Acerca del caso francés, Francois de Dainville, L'éducation des jésuits (XVI-XVII siècles), París: Les Editores de Minuit, 1978, "L'enseignement scientifique dans les collèges des jésuits au dix-huitième siècle».

4 Concentrada exclusivamente en el mundo inorgánico. 
contenido se publicaría con el título An introduction to Natural Philosophy: or Philosophical Lectures Read in the University of Oxford, Ann. Dom. 1700. To which are added, the Demonstrations of Monsieur Huygen's Theorems, concerning the Centrifugal Force and Circular Motion ${ }^{5}$ (Londres, 1720). Posteriormente en 1710 continuaría estas labores J. T. Desaguliers, quien dio el paso definitivo en la definición de esta disciplina mediante la redacción de $A$ Course of $E x$ perimental Philosophy (Londres, 1734-44).

Estas mismas prácticas se extenderían tanto a otras ciudades inglesas como a otros países europeos, como Francia, Holanda, Italia, Suiza, Portugal, Escocia, Alemania y España. Sin embargo, en cada uno de estos lugares la enseñanza de esta disciplina adquirió diferentes matices. Por ejemplo, en el caso de Francia hay que tener en cuenta que existía una firme tradición de apoyo a las ideas cartesianas. A pesar de existir algunos precedentes aislados, Newton comenzó a ser admitido en el país galo a partir de finales de la década de $1730^{6}$. Estas circunstancias influyeron sin duda en uno de los principales difusores de la física experimental en el país galo: J. A. Nollet. Después de realizar un viaje por Inglaterra y Holanda, el autor francés iniciaría un curso de física basado en la demostración experimental. Si bien los contenidos no difieren sustancialmente con respecto a las exposiciones de autores ingleses $\mathrm{u}$ holandeses, algunos principios, mencionados $\mathrm{y}$ empleados por ejemplo en las Leçons de physique (París, 1743-48), significaron un claro distanciamiento de las posturas defendidas por esos personajes. Así, para Nollet era fundamental no presentarse bajo los auspicios de filósofo alguno, ya fuera Descartes, Newton o Leibniz, autores que admira pero que no sigue en las exposiciones estrictamente experimentales de su obra ${ }^{7}$. En su teorla de la materia, en cambio, sigue esencialmente las ideas cartesianas cuando ofrece explicaciones de los fenómenos gravitatorios, eléctricos o magnéticos. Por otra parte, a pesar de aceptar en el prólogo de Leçons una cierta utilidad al álgebra y a la geometría ${ }^{8}$, no aparece un solo ejemplo de uso de las matemáticas en su obra (circunstancia que le distancia de las prácticas de, por ejemplo, W. J. 'sGravesande, uno de los grandes físicos experimentales holandeses). Estas son las ideas que Nollet pondría

5 Traducción de Introductio ad veram physicam, 1701.

6 El tema de la difusión del newtonismo en Francia ha sido estudiado por P. BRUNET, Lintroduction des theories de Newton en France au XVIII siecle, París, 1931 y por H. Guerlac en los trabajos reunidos en la obra Newton on the Continent, opus cit.

7 Leçons, París, 1775, 8. $^{2}$ ed., vol. I, págs. XX-XXI.

8 Ibid., págs. XVIII-XIX. 
en práctica en los cursos impartidos en el Collége de Navarre, adscrito a la Universidad de París ${ }^{9}$, y en las escuelas de la Fére y Mezières.

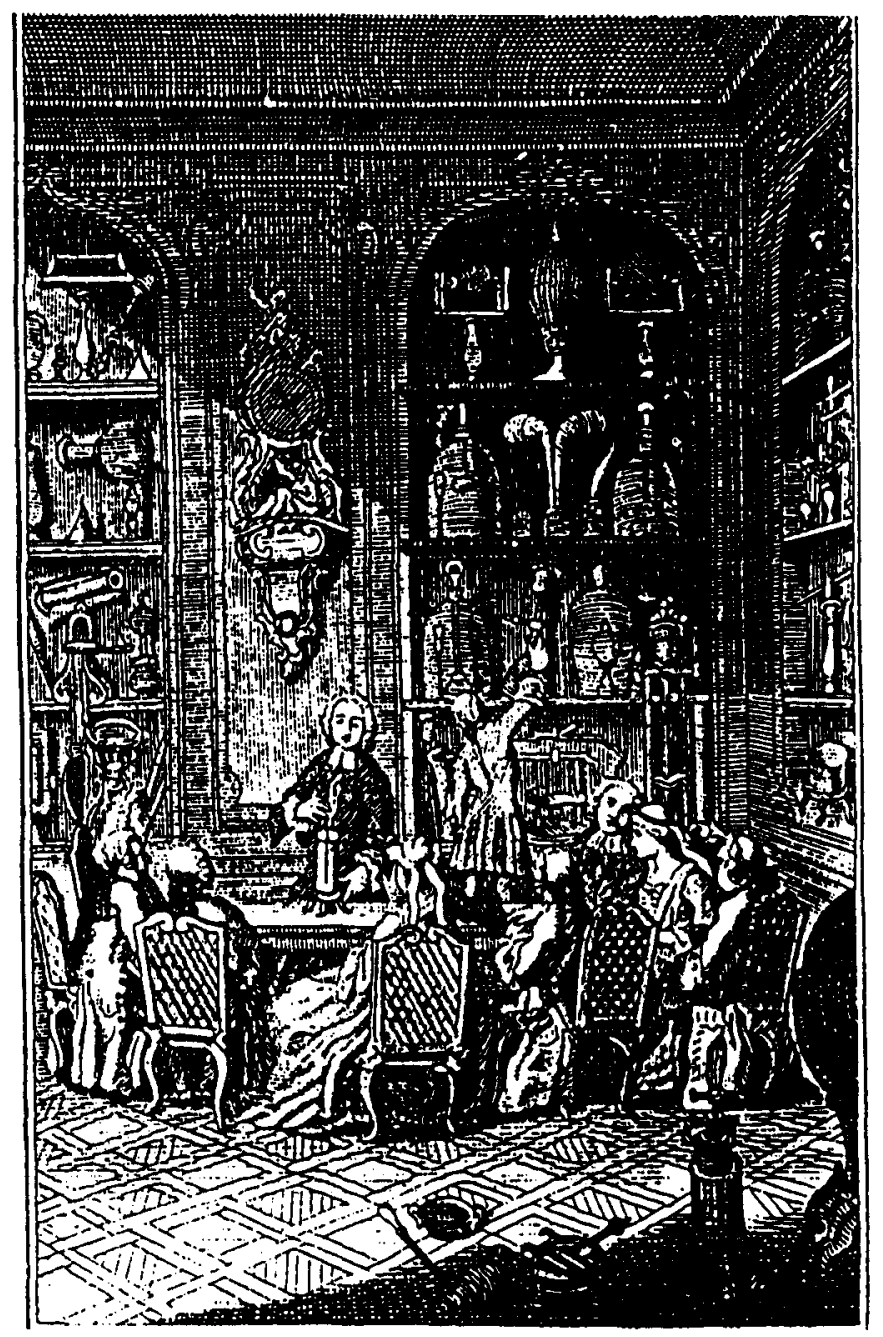

[Fig.1.] Demostración experimental, acompañada de los aparatos empleados habitualmente en este tipo de lecciones, seguin Nollet, Leçons de physique.

9 Primer curso de estas características impartido en una dependencia universitaria, J. L. HeIlbron, "J. A. Nollet", Ch. C. (ed.), Dictionary of Scientific Biography, Nueva York: Scribner \& Son, 1970, vol. 10, pág. 145. 
La introducción de la física experimental en las aulas universitarias holandesas mantiene algunas ligeras diferencias con el caso francés. En ambos países encontramos centros en los que se produce una lenta transición del artistotelismo al cartesianismo, pero difieren en el modelo educativo que debía sustituir al inspirado en este último. Por ejemplo, en la universidad de Leiden, W. Sanguerdius (16641724 ) inició en 1669 sus clases de filosofia natural con unos planteamientos que le valieron algunas enemistades con los responsables, de corte cartesiano, de la asignatura ${ }^{10}$. En ese momento ocupaba el puesto de lector, pero en 1705 , cuando finalmente fue nombrado profesor de la disciplina de fisica, se produjo un importante desarrollo de las demostraciones experimentales, que Sanguerdius llevaba a cabo especialmente en su casa. Para este tipo de demostraciones ideó una máquina pneumática, que aún se conserva en el Museo Boerhaave de Leiden ${ }^{11}$. Después de su muerte, la física cayó en letargo hasta la llegada del citado 's Gravesande.

W. J. 'sGravesande habia sido alumno de $H$. Boerhaave, quien a su vez era profesor de medicina y botánica, y posteriormente, de química en la Facultad de Medicina de esta misma universidad. Esta circunstancia no debe pasar desapercibida, ya que fue precisamente Boerhaave uno de los primeros en Leiden que aceptaron los procedimientos asociados con los trabajos cientificos de Newton, además de expresar su admiración por la escuela británica de filosofía natural ${ }^{12}$. Así, 'sGravesande no sólo recibiría esta influencia, aceptando abiertamente el newtonianismo (su obra más importante lleva por título Physices elementa mathematica, experimentis confirmata. Sive, introductio ad Philosophiam Newtonian), sino que mostraría un notable interés por las matemáticas y su aplicación en la física. Precisamente fueron las matemáticas la disciplina que más le atrajo en su aproximación autodidacta a la ciencia, ocupación que compartía con sus estudios de leyes en la propia universidad de Leiden. Después de graduarse inició la carrera profesional en La Haya, ciudad en la que fue coeditor del Journal Littéraire (1713),

10 Acerca de la incorporación de las ciencias a las universidades holandesas y, en general, sobre la ciencia desarrollada en este pals en los ss. XVII y XVIII, K. van BERKEL, "Academics and Aristocrats, 1620-1700" $y$ "Science in the Service of the Enlightenment, 1700-1795", en K. van BERKEL, A. van HELDEN y L. PALM, A History of Science in the Netherlands, Leiden: Brill, 1999, págs. 37-94.

11 Sobre los primeros gabinetes demostrativos de Leiden y las máquinas que actualmente se conservan, P. de CleRCQ, The Leiden Cabinet of Physics, Leiden: Museum Boerhaave, 1989, págs. 4-12.

12 E. G. Ruestow, Physics at $17^{\text {th }}$ and $18^{\text {th }}$ Century Leiden: Philosophy and the New Science in the University, La Haya: Martinus Nijhoff, 1973, pág. 115. 
donde publicaría diversos artículos y recensiones cientificas ${ }^{13}$. En 1715 'sGravesande viajó a Inglaterra como representante del gobierno holandés en la coronación de Jorge I, circunstancia que aprovechó para permanecer en ese pals durante dos años y entrar en contacto con el ya mencionado Desaguliers. Gracias a los comentarios favorables de Newton, al que también conocería, el autor holandés fue recomendado como profesor de astronomía y matemáticas en la Universidad de Leiden, puesto que consiguió en 1717. Posteriormente comenzaría sus propias lecciones de fisica experimental, aceptando el puesto de Sanguerdius después del fallecimiento de éste. Su obra más importante es la referida anteriormente Physices elementa mathematica (Leiden, 1720 y 1721), que fue acogida favorablemente en Inglaterra, Italia, y como veremos más adelante, España.

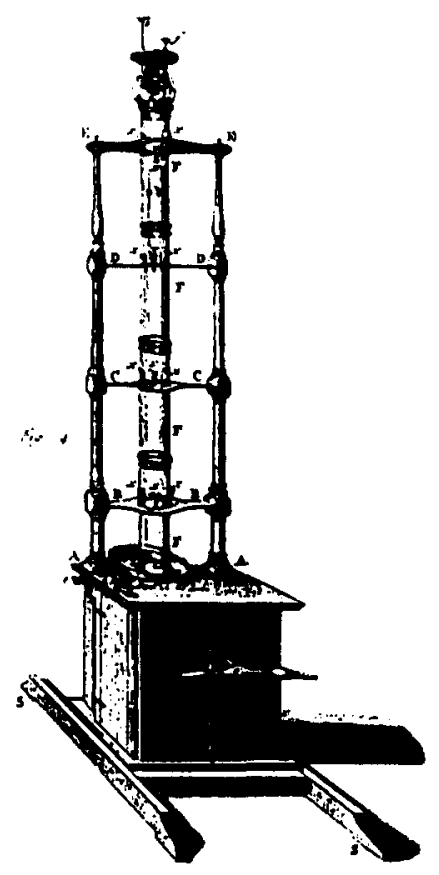

[Fig.2.] Tubo de Newton, ajustado en una maquina pneumdtica, empleado en las demostraciones de caida de cuerpos de diferente naturaleza en el vacio, según'sGravesande, Physices elementa.

13 Recogidos en W. 's Gravesande, Ouvres philosophiques et mathematiques, ed. de J. N. S. Allamand, Amsterdam, 1774. 
Otro de los grandes personajes asociados con la renovación de la física y con la difusión de la física experimental es Petrus van Musschenbroek, sucesor de 'sGravesande y como éste, discípulo de Boerhaave. También viajó a Londres, donde se familiarizó con el ambiente científico de la capital inglesa. A su vuelta comenzó a practicar la medicina (el doctorado en esta disciplina lo habia conseguido en 1715). Después de doctorarse también en filosofía y de pasar por las universidades de Duisburg (Alemania) y de Utrecht (Holanda), donde alcanzaría una gran fama como físico, entró en la Universidad de Leiden, ocupándose de la enseñanza de las matemáticas, de la filosofía y de la física experimental hasta su fallecimiento en 1761. Su producción, de gran influencia en diferentes centros europeos, es extensa, destacando Essai de Physique (Leiden, 1739), Elementa physicae (Leiden, 1741), traducida al inglés en $1744 \mathrm{y}$ al alemán en 1747, y Cours de physique expérimentale et mathématique (París, 1769), traducción al francés de la Introductio ad philosophiam naturalem (Lugduni Batavorum, 1762), el más importante de los trabajos del autor holandés.

La física experimental defendida por estos autores aspiraba a convertirse en una alternativa programática tanto del aristotelismo como del cartesianismo ${ }^{14}$. En el siglo XVII, la filosofía de Descartes había respondido a una vieja aspiración consistente en ofrecer una completa comprensión de la naturaleza a partir del establecimiento de las causas naturales fundamentales. Además, dentro de las universidades, el viejo estilo estaba representado por la perpetuación de los sistemas filosóficos, acompañados por apéndices relacionados con la física que se ajustaban a los métodos pedagógicos expositivos. Lo que pretendía la pareja holandesa, por el contrario, era, por un lado, eliminar las explicaciones basadas en causas últimas, y por otro, desarrollar un tipo de enseñanza donde el alcance de la física no estuviera determinado por su posición en el seno de un sistema filosófico, sino por las prácticas de las investigaciones experimentales. Ideas que encontraron una respuesta real, si bien excepcional, en los curricula filosóficos; por ejemplo, a partir de 1730 en las universidades protestantes alemanas, la cátedra de física llegó a ser una aspiración en sí misma, no un medio para continuar la carrera médica o eclesiástica ${ }^{15}$. Igualmente, en Leiden, la física llegó a tener una categoría similar ${ }^{16}$.

14 Sobre este aspecto, Ruestow, "sGravesande and Musschenbroek: Newtonianism at Leiden" y "Conclusion: Science, Philosophy and Pedagogy", opus cit., págs. 113-154.

15 J. L. HeIlbron, "Experimental Natural Philosophy», en G. S. Rousseau \& R. PORTER, The Ferment of Knowledge. Studies in the Historiography of the Eighteenth Century Science, Cambridge: Cambridge University Press, 1980, pág. 376.

16 RUESTOW, opus cit, pág. 150. 
Junto a este distanciamiento con respecto a las viejas posturas, 'sGravesande y Musschenbroek interpretaron la fisica experimental de una manera sensiblemente diferente a la de algunos de sus contemporáneos, en particular a la de su colega francés Nollet. La principal discrepancia se refería a la teoría de la materia defendida por ambos. Como ya hemos visto, los autores holandeses admitían el punto de partida newtoniano, lo que significaba en su caso defender la existencia de fuerzas y del vacío, algo con lo que el autor de las Leçons no comulgaría. Al mismo tiempo, las fuerzas significaban algo determinado dentro de este ambiente experimentalista, en particular en el caso de Musschenbroek. Según este último, hay fenómenos cuya explicación completa (es decir, a partir de su naturaleza) resulta imposible, como la gravitación, los fenómenos de atracción y repulsión (que explican la capilaridad, algunos efectos químicos y el magnetismo) y, sobre todo, el concepto de fuerza. Por tanto, lo más lejos que podemos llegar es a la determinación de las leyes o regularidades de los fenómenos mediante una atención paciente a los efectos observados.

En conclusión, reuniendo las diferentes propuestas vistas, podemos hacernos una imagen global de las pretensiones de los físicos experimentales. Por un lado, la explicación de los fenómenos debía reducirse a la concepción de un mecanismo que se ilustrase mediante una demostración experimental (o analogia), o bien sus consecuencias tendrían que comprobarse mediante un experimento. Por otro lado, quienes desconfiaban de los mecanismos, preferían centrarse en las regularidades observadas a partir del efecto global provocado por un cuerpo en otro e intentar llegar, en función de estos datos, a una ley simple empleando una proporción. Estos principios eran aplicados, sobre todo, en el estudio del calor, el magnetismo y la electricidad, disciplina, esta última, cuyo desarrollo, especialmente a partir de la segunda mitad del siglo XVIII, reforzó aún más las posturas anteriores. Es cierto que existieron otros filósofos naturales que mediante el experimento realizaron importantes contribuciones a la física del siglo ilustrado. Tenemos, por ejemplo, los casos de T. Aepinus, $\mathrm{Ch}$. Coulomb o H. Cavendish. Sin embargo, su forma de tratar los problemas, firmemente inspirada en el uso de las matemáticas, y la propia concepción que tienen del experimento les separaron de los personajes que se han tratado anteriormente. Por eso, este grupo, que cuenta con su propia tradición, debería más propiamente recibir la denominación de "ffisicos experimentales matemáticos».

Se han citado las corrientes más importantes de la física experimental (en sentido estricto) que se gestaron en Francia y en Holanda. A partir de aquí se extendería por diversos países europeos. En Inglaterra, la obra de los holandeses 
encontró un suelo fértil. Así, de la obra de 'sGravesande se realizaron inmediatamente traducciones al inglés (en concreto, la preparada por Desaguliers conoció 6 ediciones). También las obras de Musschenbroek se tradujeron al inglés, francés, alemán, sueco y holandés. Por otra parte, en Escocia, el Dr. Demainbray inició un curso en 1740 en Edimburgo según el patrón de Desaguliers y 'sGravesande ${ }^{17}$. Así mismo se establecerían cátedras de esta disciplina en Glasgow (1727), St. Andrews (1747) y Aberdeen (1753).

En Italia la institucionalización de la física experimental comenzó realmente a finales de la década de los años 30 , ya que hasta entonces la filosofia seguia en manos de clérigos, cuyos programas contenían mezclas oportunas de Aristóteles y Descartes. En la universidad de Bolonia contaban con un profesor de física en 1737 y el Istituto de esta ciudad adquirió, gracias a la mediación del papa Benedicto XIV, una colección de aparatos basada en el diseño de 'sGravesande ${ }^{18}$. En Padua (donde se encontraba G. Poleni, que también era profesor de matemáticas) la física experimental se enseñó desde 1739; en Roma, en la Sapienza, contaron entre 1744 y 1746 con una cátedra de filosofía racional y experimental. A esta relación se sumarían las siguientes ciudades con cátedras de física experimental: Pisa, Turín, Perugia, Sassari, Pavía, Módena, Parma y Nápoles.

En Suiza contaron con una cátedra de física experimental en la Académie de Genève, que estuvo a cargo de J. Jallabert a partir de 1737. Éste habla viajado por Inglaterra, Holanda y Francia, conociendo a Musschenbroek y Nollet y adquiriendo diversos aparatos para las demostraciones.

A esta relación de ciudades se suma Coimbra, en Portugal, sobre cuya universidad contamos con extensa información ${ }^{19}$. A partir de 1772 se adoptaron medidas destinadas a su renovación, que comenzaron por sustituir la anticuada Facultad de Artes por la de Filosofía, donde se impartirían las enseñanzas de filosoffa racional y moral, historia natural, física experimental y química teórica y

17 M. DaUmas, Les instruments scientifiques aux XVII et XVIII' siècles, París: P. U. F., 1953, pág. 182.

18 Los detalles sobre las distintas máquinas adquiridas así como de su finalidad se encuentran en la tesis lelda por Stefano Belli en Bolonia, Le "camere" di fisica dell'Istituto delle Scienze di Bologna (1711-1758), 1994.

${ }_{19}$ Sobre este tema, Rómulo de Carvalho, Historia de Gabinete da Universidad de Coimbra, Coimbra: Universidade de Coimbra, 1978; en general sobre la física experimental en Portugal, del mismo autor, $A$ fisica experimental em Portugal no séc. XVIII, Lisboa: Instituto de Cultura e Lingua Portuguesa, 1982. 
práctica. Los artículos de los estatutos de la Universidad especificaban, en particular para la física experimental, que se animase al cuestionamiento de la física cartesiana. Por otra parte, se aconsejaba el uso de la conjetura y la analogía (pilares básicos de la nueva lógica adaptada a los requisitos científicos), que han conducido, según se asegura, a realizar importantes descubrimientos. Mediante estos estudios se pretendía igualmente difundir y afianzar una metodología basada en la experiencia entre el público asistente. Para esta finalidad se organizarán las lecciones en la "Casa de las máquinas», procurando que los alumnos no fueran meros espectadores, sino que trabajaran e hicieran por sí mismos las experiencias. Alumnos que se dedicarían a la física experimental en el tercer año del curso filosófico, una vez superadas la filosofia racional, la historia natural y la geometrí.

A pesar de los excelentes medios y cuidadosos planteamientos iniciales, los cursos de física experimental despertaron un escaso entusiasmo entre el publico portugués. En los cinco primeros años asistieron solamente cuatro alumnos correspondientes a los matriculados en el curso filosófico. A este grupo, en cualquier caso, habría que añadir los procedentes de las facultades de matemáticas y medicina, cuyo curriculum también incluía la física experimental.

En general, para su puesta en práctica, la enseñanza de la física experimental exigfa contar con un importante presupuesto. A los gastos relacionados con el sueldo del profesor y la provisión de una adecuada sala de demostraciones, se sumaban los propios de la adquisición de una colección de aparatos ajustada al programa de la asignatura y la contratación de un ayudante, normalmente un mecánico, encargado de preparar y mantener los instrumentos. Con respecto a las universidades, en las protestantes era el profesor el encargado de adquirir y mantener las piezas del gabinete. En las católicas, la reunión del utillaje demostrativo se lograba a través de fondos propios del centro o por medio de subvenciones estatales o particulares. A pesar de que en un principio estos aparatos tenían una finalidad educativa, en un número limitado de universidades fueron encargadas piezas destinadas a la investigación. En este último caso se encuentran Halle, Göttingen, Pavla (impulsado por los estudios de Volta a partir de 1777), Edimburgo (después de la 2. ${ }^{2}$ mitad del siglo XVIII) ${ }^{20}$, y a finales del setecientos, Coimbra ${ }^{21}$.

20 RUESTOW, opus cit., págs. 152-153.

21 Les mécanismes du génie. Instruments scientifiques du XVIILe et XIX siècles. Collection du Cabinet de Physique et de L'Observatoire Astronomique de l'Universite de Coimbra, Charleroi, Palais de Beaux-Artes, 21 de Septembre-22 de Décembre, 1991, pág. 65. 
Hasta ahora no se ha hecho ninguna referencia al caso español debido a que, por su extensión, se reservará un apartado para su consideración detallada al final del artículo. Seguidamente, con el fin de continuar con la descripción de los procedimientos didácticos empleados en general en Europa, se atenderá a los contenidos que formaban un programa y a los métodos empleados para su instrucción.

Programas y colecciones de máquinas y aparatos

El programa de la disciplina dependía del manual elegido. Ya se ha visto que los autores preferentes en la elección de los contenidos eran los holandeses, hecho que no significa que para cuestiones particulares, ya fuera para la construcción de un aparato o para la realización y comentario de alguna experiencia, se acudiera a otros textos. Para la exposición de los apartados de esta disciplina, por tanto, se tendrá en cuenta la distribución temática de la obra de Musschenbroek ${ }^{22}$. Varias razones justifican esta elección. En primer lugar, su producción reúne las innovaciones de las tradiciones continental e inglesa; segundo, su obra se enmarca entre 1730 y 1765 , recogiendo las aportaciones de la primera mitad del siglo y anunciando las tendencias de la segunda. Otro elemento importante es la amplia difusión de sus escritos, que circularon por Espana, Portugal, Alemania, Inglaterra, Italia, Francia, y por supuesto, los Países Bajos. Y, por último, mantiene una posición característica de la época en relación con el uso de la experimentación y de las matemáticas en la física.

De acuerdo con los manuales de Musschenbroek, la distribución de los temas que configuran el ámbito de desarrollo de la física experimental es la siguiente $^{23 .}$

1. PROPIEDADES DE LOS CUERPOS. Consideraciones generales.

2. MECANICA. Cinemática, dinámica, percusión; máquinas, fricción; movimiento compuesto, péndulos, proyectiles; colisiones; resistencia de materiales.

22 Como aconseja Heilbron, opus cit, 363.

23 HeIlbRON, «Experimental..", opus cit., págs. 366-7. 
3. MECANICA DE FLUIDOS. Tensión superficial, capilaridad; hidrostática, gravedad específica; higrometría; flujo de líquidos.

4. AGUA. Propiedades físicas; gravedad especifica; congelación, ebullición, evaporación.

5. LUZ. Optica geométrica; colores, anillos de Newton; el ojo, instrumentos ópticos; luminiscencia.

6. AIRE. Gravedad específica, densidad; instrumentos pneumáticos; relaciones entre volumen y presión; sonido.

7. FUEGO. Pirómetros; espejos ustorios y lentes; termómetros; temperaturas de fusión; índices de absorción de calor; calorimetría; naturaleza de la llama, el calor y el fuego.

8. ELECTRICIDAD.

9. MAGNETISMO.

10. METEOROLOGIA. Formación de nubes, lluvia, granizo, nieve, rocío; arco iris, coronas, halos, parhelia; auroras, luz zodiacal, fuego lambente, relámpagos; vientos.

Los distintos saberes aquí enumerados, y algunos más que configuran la física, se distribuyen, según las concepciones generales de Musschenbroek, de acuerdo con las siguientes categorías:

Física experimental (cuerpos en general):

La parte general de la física experimental está basada en las propiedades generales de $1 .^{2}$ y $2 .^{2}$ clase ${ }^{24}$, admitiendo la aplicación de las matemáticas. Las propiedades de primera clase comprenden, a su vez, la extensión, la impenetrabilidad, la fuerza de inercia, la movilidad, la reposabilidad y la figurabilidad, y las de segunda clase, la gravedad y la fuerza de atracción. En definitiva, todas las propiedades que afectan a la materia ordinaria.

Así, esta parte de la física comprende las siguientes ramas:

- Mecánica elemental

24 P. VAN MUSSCHENBROEK, Essai de physique, Leiden, 1751 (1.2 ed. 1736-39), págs. 22 23. 
- Hidrostática elemental

- Hidrodinámica elemental

- Adherencia 25

- Magnetismo 26

La física particular, por su parte, está basada en las propiedades no comunes a todos los cuerpos. En este caso nos encontramos con una larga lista en la que aparecen referencias al calor, el sonido, la transparencia, la solidez, etc. Aquí, por tanto, se incluyen los siguientes estudios:

- Electricidad

- Agua

- Fuego

- Aire

- Luz

Meteorología (fenómenos atmosféricos)

- Meteoros aéreos

- Meteoros acuosos

- Meteoros ígneos

Historia natural (cuerpos terrestres)

Centrándonos ahora en la física experimental, las propiedades generales que comprende admiten el aumento y la disminución, por lo que posibilitan

25 La adherencia se incluye en el primer grupo, así como también el magnetismo, porque dependen de la materia ordinaria; en los otros casos sería necesario un fluido especial. Sobre los trabajos experimentales realizados en el primero de estos ámbitos, que presuponen la existencia de fuerzas a corta distancia, E. C. Millington, "Capillarity and Cohesion in the Eighteenth Century", Annals of Science, 5 (1947): 352-369.

26 En realidad, en este caso, Musschenbroek representa una excepción, ya que la tendencia dominante durante todo el siglo dentro de los físicos experimentales fue el apoyo con más o menos matices de la "teoría de la circulación", inspirada en Descartes; por tanto, estos estudios más bien deberian incluirse dentro de la física particular. Acerca de esta cuestión, R. W. HoME, "Magnetism", en T. A. Aepinus, Essay on the Theory of Electricity and Magnetism, introd. y notas de R. W. Home, Princeton: Princeton University Press, 1979, págs. 137-188, espec. págs. 162-163. 
la aplicación de las matemáticas. En segundo lugar, la división entre la parte general y la particular de la física experimental no expresa una distancia infranqueable ${ }^{27}$ : una parte importante de las áreas contempladas en la física particular se encuentran conectadas con la general (por ejemplo, el estudio de las propiedades del agua o del aire derivadas de su peso). Además, al considerar la virtud atractiva como una propiedad general, cuyo estudio podía acometerse considerando sus efectos y sus leyes, algunos campos (electricidad y magnetismo) relegados a estudios descriptivos propios de las historias naturales podrian consolidarse como disciplinas características de la física general. Por su parte, la meteorología, en cuanto que examina fenómenos vinculados con las propiedades del aire, del agua o de la electricidad puede remitirse a las conclusiones establecidas en la física particular o también en la general. Según este esquema, la física se distingue, por un lado, de la química en cuanto que a la segunda están reservadas aquellas propiedades particulares que no se remiten a ninguna de las generales ${ }^{28}$, y por el otro, de las matemáticas mix$\operatorname{tas}^{29}$ en que éstas solamente se ocupan de los cálculos prácticos derivados de las propiedades de primera clase.

27 De hecho, en los textos de Musschenbroek no se reconoce explícitamente esta división dentro de la disciplina, solamente se establece en las propiedades (generales o particulares) que trata la física.

28 G. F. VENEL, en su artículo "Chymie" de la Encyclopédie (París, 1751, vol. 14), refuerza ese apreciación de distanciamiento entre la física y la química. Aquí apunta que la primera disciplina está destinada al estudio de las propiedades mecánicas de los cuerpos, fundadas en la masa, la presión, el peso, la fuerza, atendiendo además exclusivamente a la figura, el tamaño, el movimiento y la posición. Frente a estas cualidades "exteriores" se encuentran las «interiores o químicas", que son las que explican la afinidad, la separación de los corpúsculos por medio del calor y la volatilidad. Sobre la emancipación de la química, M. CrosLAND, "Chemistry and the Chemical Revolution», G. S. ROUSSEAU \& R. PORTER, The Ferment of Knowledge. Studies in the Historiography of Eigtheenth-Century Science, Cambridge: Cambridge University Press, 1980, 395-396.

29 En los siglos XVI, XVII y parte del XVIII se establecía una distinción entre matemáticas puras, que comprendían la geometría y la aritmética, y matemáticas mixtas, que incluían astronomía, óptica, estática, topografía, navegación, fortificación y artillería, entre otras. Aristóteles ya había destacado esta diferencia entre matemáticas puras y matemáticas "más próximas a la física», como la óptica, la armonia y la astronomía (Fisica, 194a, 7-12; Madrid: Gredos, 1995, págs. 136-137); en la Metafísica incluye a la mecánica (1078a 14-17; Madrid: Gredos, 1994, pág. 513). Ahora bien, según el filósofo griego, aunque la matemática se interese, por ejemplo, por la figura de la Luna y el Sol, que son también competencia del físico, no las considera en tanto límites de un cuerpo físico, "ni tampoco estudia los atributos mencionados en tanto que atributos de tales cuerpos. Por eso también los separan, pues por el pensamiento se los puede separar del movimiento» (Física, 193b, 33-35; opus cit., pág. 136). 


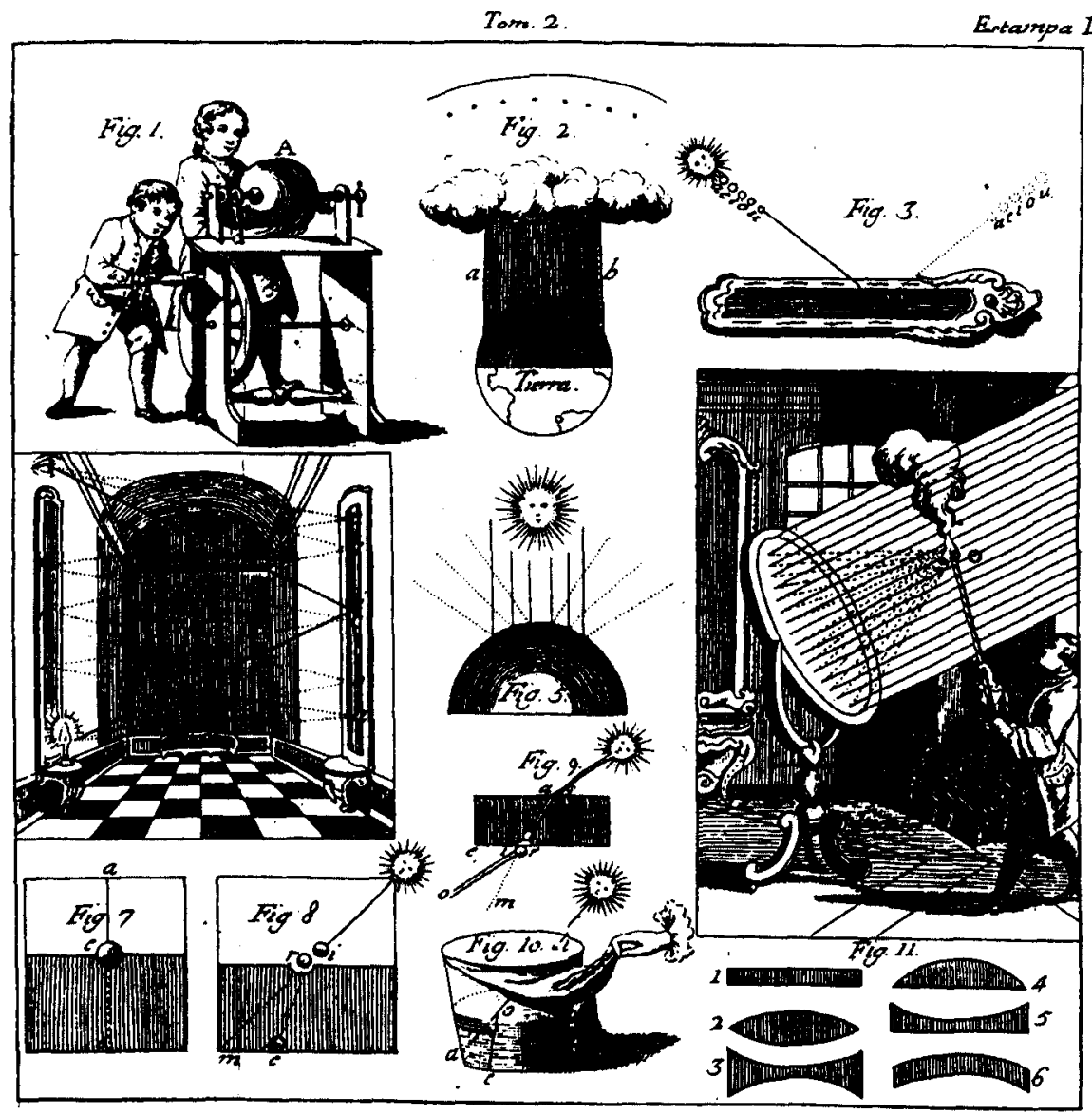

[Fig. 3]. Demostraciones relacionadas con la electricidad, bptica y calor, según Teodoro de Almeida ${ }^{30}$, Recreación filosófica o dialogo sobre la filosofla natural para la instrucción de personas curiosas que no frecuentan las aulas, Madrid, 1786.

Prácticamente todos los temas enumerados, así como sus respectivos apartados, estaban acompañados de un aparato que ilustrase o confirmase las afirmaciones que contenían. Para ello, como ya se ha dicho, era necesario disponer de una "Sala de Máquinas" o gabinete, elemento que completaba las exigencias de una física experimental cuyo fundamento y justificación era la reproducibilidad

30 Autor portugués nacido en 1722 y dedicado a la enseñanza de la física experimental. 
de los procesos naturales mediante el utillaje demostrativo ${ }^{31}$. Si abrimos un manual cualquiera, observaremos detalladas descripciones de las condiciones del experimento, de los aparatos empleados, del desarrollo de la experiencias y de los efectos observados. También en muchas ocasiones encontramos largas exposiciones, acompañadas de tablas de datos, exentas de una conclusión. Para este cometido, las universidades y escuelas adquirían o encargaban la fabricación de costosas piezas. Como los manuales empleados se repetían en los centros, los instrumentos que se encuentran en los diferentes gabinetes europeos también, solamente diferenciándose en detalles locales de estilo. Daumas resume con estas palabras la uniformidad del utillaje demostrativo:

Dentro de todas las colecciones encontramos juntos dos aparatos esenciales: la máquina eléctrica y la bomba pneumática. Hacia finales de siglo, la moda de los aparatos de demostración del estilo 'sGravesande se había debilitado; los amateurs habían dirigido su curiosidad hacia la máquina eléctrica y los accesorios que la acompañaban: conductores, excitadores, botellas de Leiden, péndulos de médula de saúco, cuadros mágicos y fuentes luminosas...La bomba pneumática gozaba entre todos de un favor general, encontrándose de una forma generalizada en todos los inventarios; se acompañaba normalmente de una bomba de compresión y de toda una serie de accesorios: platinas, campanas, tubos barométricos, hemisferios....El microscopio, el telescopio, el anteojo astronómico, a menudo de origen inglés, los barómetros y termómetros de salón, la marmita de Papin son los objetos que normalmente se encuentran acompañando a la máquina eléctrica y la pneumática ${ }^{32}$.

31 Acerca de los gabinetes de instrumentos cientificos y, en general, sobre la fabricación de este tipo de útiles, véase Daumas, opus cit., págs. 324-385; por países, en Escocia, CLARKE et al., Brass and Glass. Scientific Instrument Making Workshops in Scotland (Edimburgo: National Museum of Scotland, 1989) y D.J. BRYDEN, Scottish Scientific Instruments (Edimburgo, 1973); en Irlanda, J. E. BURNETT y A. D. MORRISON-LOW, Vulgar and Mechanick. The scientific Instrument Trade in Ireland (1650-1912) (Dublín: Royal Dublin Society, 1989); en los Paises Bajos, CROMmelin, Descriptive Catalogue of the Physical Instruments of the 18th Century in the Rijksmuseum voor de Geschiedemir der Natuurwetenschappen (Leiden: National Museum of the History of Science, 1951) y Peter de CleRCQ "Science at Court: The Eighteenth-Century Cabinet of Scientific instruments and Models of the Dutch Stadholders", Annals of Science, 45 (1988), 113-152; en los Estados Unidos, Silvio A. BEDINI, Early American Scientific Instruments and their makers. Washington: United States National Museum Bulletin, 231 (1964); en Alemania, G. F. Brander: Alto Brachner et al., G.F. Brander (1713-1783): Wissenschafliche Instrument aus seiner Werkstaff. Munich: Deutsches Museum, 1983.

32 Daumas, opus cit., pág. 193. 
El número de piezas variaba de un gabinete a otro en función del presupuesto, de las ayudas recibidas y de su carácter estatal o no (los primeros normalmente contaban con más fondos). Por ejemplo, entre los más numerosos se encontraba el de Coimbra con aproximadamente 500 aparatos a finales de siglo. También el perteneciente a la Universidad de Padua, con 392 en el año 1764 , entraba en la categoría de los importantes ${ }^{33}$. Entre los particulares, el más destacado es el del físico Alexander Charles, en Francia, con 330 piezas. Siguiendo en el país galo, en sus provincias existían diferentes colleges, cuyos gabinetes sobrepasaban el centenar de instrumentos, como el de Duai y el de Vendome; el resto posé́a dotaciones más modestas, entre 25 y 40 piezas, como el de Moulins, Pontlevoy y Seminario de Puy ${ }^{34}$.

Si bien entre estos gabinetes, considerados en su conjunto, se aprecia una notable homogeneidad, dentro de las colecciones sí es posible detectar diferencias importantes entre unos aparatos y otros. Estas particularidades nos informan sobre el tipo de física que se practicaba en la época. Así, tomando como referencia el programa expuesto anteriormente, en las secciones dedicadas a la mecánica aparece un amplio conjunto de piezas destinadas, en un caso, a las demostraciones relacionadas con leyes clásicas de esta parte de la física, y en otros, a la exposición del funcionamiento de máquinas. El primer ámbito no contenía aportaciones relevantes (se trataba simplemente de corroborar leyes ya establecidas en el siglo XVII) excepto en aquellos casos en los que se incorporaban piezas que reproducian debates vigentes, al menos, en la primera parte del siglo XVIII, como los referentes a cuál era la adecuada medida de la fuerza, mv o $\mathrm{mv}^{2}$. En cuanto al segundo (funcionamiento de máquinas), es especialmente novedosa la inclusión de aparatos dentro de una variada colección de palancas, grúas, cabrestantes, etc. cuyo cometido era estudiar las condiciones reales de funcionamiento de estos ingenios, es decir, aquéllas en las que se tienen en cuentan tensiones, rozamientos y resistencias entre los elementos que componen estas máquinas. Estos trabajos darían lugar a la mecánica práctica, que conoció un importante desarrollo durante el siglo XVIII ${ }^{35}$.

33 J. L. HeIlbron, Electricity in the $17^{\text {th }}$ and $18^{\text {th }}$ Centuries. A study of early physics, Berkeley: University of California Press, 1979, pág. 142.

34 DAUMAS, opus cit., pág. 193

35 Sobre algunos aspectos y resultados de esta vertiente de la mecánica, A. BARACCA, "El nacimiento y desarrollo de los conceptos de trabajo y energía en el contexto de la mecánica práctica durante la Primera Revolución Industrial», en A. Elena, J. OrdoñEz y M. Colubi (Comps.), 
Junto a este utillaje se encontraban aquellos aparatos e instrumentos asociados no con diversos aspectos de las ciencias clásicas, sino con apartados propios de lo que en la historiografia se ha conocido como "ciencias experimentales", que correspondían a los saberes que aún permanecían en un período de formación y no disponían de teorías maduras como en el caso anterior. Dentro del programa estas ciencias formaban la física particular (debido a que en esta parte se analizaban propiedades particulares), denominación que contiene reminiscencias aristotélicas. En concreto, se trataba del calor, parte de la óptica, el magnetismo, la electricidad y el aire. En cuanto a las piezas, las colecciones disponían de instrumentos destinados a la observación, a la medición, a la producción de fenómenos y al estudio de propiedades. Algunas, cuyas características se verán a continuación, reflejan especialmente los métodos y objetivos de la física experimental.

En este grupo, los más clásicos, en correspondencia con el grado de desarrollo de sus respectivas ramas científicas, eran los de óptica y algunos vinculados con la pneumática. Así, para estas secciones se podían adquirir aparatos para mostrar las leyes de la reflexión y de la refracción (así como las combinaciones de éstas), como lentes, microscopios, telescopios, reflectómetros y refractómetros. Entre este material, no obstante, destaca la presencia de rejillas regulables para poner de manifiesto efectos de difracción, los cuales formaban parte de investigaciones desarrolladas en el s. XVIII. Con respecto a la pneumática, el aparato más destacado, presente en todos los gabinetes, como decía Daumas, era la bomba pneumática o de vacío. Con este tipo de utillaje era posible crear condiciones extraordinarias de laboratorio, y así analizar qué efectos podían observarse cuando se intentaba la transmisión del sonido o la electricidad. Para estas finalidades la obsesión de los fabricantes fue la mejora de los sistemas de válvulas y ajustes con la intención de evitar escapes o entradas de aire. Con estos perfeccionamientos se pasó de conseguir una presión de $1 / 40$ o $1 / 50$, a principios de siglo, a $1 / 165$ (modelos franceses) o $1 / 300$ y $1 / 600$ en 6 minutos de funcionamiento con los modelos ingleses fabricados por E. Nairne ${ }^{36}$.

Despues de Newton: ciencia y sociedad en la Primera Revolucion Industrial, Barcelona: Anthropos, 1998, págs. 119-146.

36 Recordemos que las máquinas de mercurio empleadas a finales del s. XIX para fabricar lámparas incandescentes alcanzaban una presión mínima de $1,3 \times 10^{-5}$ atm. y que las modernas más sofisticadas llegan hasta $1,3 \times 10^{-13}$ atm., Mara MiniaTI (ed.), Museo di Storia della Scienza. Catalogo, Florencia: Istituto e Museo di Storia della Scienza, 1997, pág. 277. 
El resto de los instrumentos, los asociados propiamente con la física experimental, tienen también un gran interés. Algunos están destinados a poner de manifiesto alguna propiedad eléctrica o magnética que hubiera representado un giro en las investigaciones llevadas a cabo en estos campos, caso en el que se encuentran la botella de Leiden o el electróforo (ambos condensadores). Este último había sido ideado por Volta en 1775 para poner en entredicho la teoría de las atmósferas eléctricas así como del fluido eléctrico. Otros aparatos, sin embargo, contenían una intención diferente: la de intentar llegar a una ley simple de la naturaleza, tal y como estaba previsto en los presupuestos de la fisica experimental. Dentro de este grupo se encontraban el dilatómetro, el electrómetro, la balanza de torsión y el calorimetro. Los dos primeros pusieron de manifiesto la ingenuidad de los filósofos naturales al pensar que la mera incorporación de una escala graduada a un aparato y la recogida de datos podía garantizar el descubrimiento de esa ley. Hecho - la concepción de una ley-que sí se produciŕa por medio del uso de la balanza (en 1785, gracias a los trabajos de Coulomb), una vez que se llevaron a cabo transformaciones conceptuales esenciales, además de las puramente experimentales, que mostraban claramente qué es lo que se estaba midiendo ${ }^{37}$.

Para llevar a cabo las demostraciones asociadas con la electricidad era fundamental contar con una máquina electrostática, otro de los elementos omnipresentes, como ya se ha dicho, en los gabinetes. Aunque existieron diversos artefactos de este tipo en las primeras décadas del siglo XVIII, es a partir de 1745 cuando se produce el verdadero despegue en su producción. Como elementos fundamentales, que formaban parte de estos generadores compactos, se encontraban el conductor principal, el colector, el cojinete y la pieza giratoria (disco, cilindro, esfera,...), normalmente de vidrio, que se sometía a rozamiento para su electrización. A partir de 1770, gracias a la introducción de diversas mejoras, se produjo un importante incremento en la carga obtenida. De los $5 \mathrm{~cm}$. de longitud de las chispas, logradas con las máquinas de 1750 (que representaban un potencial de 70.000 voltios) se pasó a los $35,5 \mathrm{~cm}$. (170.000 voltios), con las máquinas de cilindro de Nairne o los $60,9 \mathrm{~cm}$. (330.000 voltios) con las máquinas de disco de J. Cuthbertson ${ }^{38}$. Con estas máquinas era posible electrocutar

37 Para Daumas, este tipo de instrumentos significaron un cambio de orientación fundamental dentro del utillaje empleado en la física, M. Daumas, "Precision of measurement and physical and chemical research in the Eighteenth Centuryn, en A. C. CrombIE (ed.), Scientific Change, Londres: Heinemann, 1963, pág. 428.

38 Datos de W. D. HACKMANN, Electricity from Glass: the History of the Frictional Electrical Machine (1600-1850), Alphen aan den Rijn: Sijthoff \& Noordhoff, 1978, pág. 16. 
animales, fundir metales y electrolizar agua. Así mismo los diferentes estudios llevados a cabo con los generadores permitieron formular las primeras relaciones entre la carga, la superficie del conductor y la intensidad.

Todas estas piezas, junto con otras, como los termómetros, barómetros e higrómetros (diseñados, una vez que su fiabilidad estaba garantizada, para la recogida de observaciones), cumplieron la misión de asegurar que las afirmaciones que componían los programas de física experimental no fueran especulaciones vacias, sino, como demandaban algunas corrientes dominantes del Siglo de las Luces, un producto de la observación, de la experiencia y del trabajo callado y rutinario del laboratorio. Tal y como estaba planteada esta disciplina, por tanto, su público potencial estaba constituido por quienes desearan obtener el título de bachiller en Artes (y estuvieran matriculados en facultades con programas renovados), y también por aspirantes a médicos, a cirujanos, a químicos, o bien por algunos artesanos y por curiosos impacientes por observar efectos espectaculares en la naturaleza. En total, por tanto, no representaban muchas personas, y su incidencia en los diferentes países dependió del desarrollo de cada uno de los colectivos mencionados.

\section{La física experimental en España}

En las primeras fases del siglo XVIII, en España la física era considerada un saber teórico y, por tanto, alejado de los planteamientos utilitaristas característicos de la ciencia española. Por esta razón difícilmente podía contar con algún apoyo estatal para su incorporación en las universidades o en otros centros de educación superior ${ }^{39}$. La única vía institucional para la entrada de los nuevos planteamientos eran las actividades pedagógicas de los jesuitas, que habían conseguido dominar una amplia red de centros de enseñanza. Dentro de este ámbito, los esfuerzos se encaminaron hacia la conciliación de las novedades con la ortodoxia aristotélica, lo que condujo hacia el mantenimiento de posiciones marcadamente eclécticas.

Con el extrañamiento de los miembros de la Compañía de Jesús (1767) comenzó a abordarse en profundidad el problema educativo. A partir de ese

39 Existe, no obstante, una excepción: la referida a la parte de la física contemplada en las matemáticas mixtas, dominadas por las ciencias mecánicas, un saber práctico, que recibieron una importante atención en los planes de estudio de las academias militares. 
momento, desde la Corona se impulsó la creación de centros donde no fuese necesaria la superposición de una estructura militar a una civil y se inició el proceso de reforma universitaria, que incluía la imprescindible unificación de los programas. $\mathrm{El}$ resultado de estas iniciativas fue el inicio de un proceso de secularización de la enseñanza y la creación de diferentes instituciones, como los colegios de cirugía, el Jardín Botánico, el Observatorio Astronómico de Madrid y los Reales Estudios de Madrid, al que me referiré en detalle más adelante. En definitiva, este hecho significó igualmente un impulso para la física experimental, que constantemente aparecía citada en los planes de estudio destinados a las universidades.

En la primera etapa destacan dos centros: el Seminario de Nobles (fundado en 1725) y el Colegio Imperial (fundado en 1624). El primero dispuso de excelentes medios para el desarrollo de las enseñanzas: se adquirió en París una amplia dotación de material (cuyo coste fue de 800.000 reales) y el profesor de física, Antonio Zacagnini, realizó una traducción de las Leçons de Nollet. Sin embargo, no parece que la física experimental despertara gran interés entre los Caballeros Seminaristas ${ }^{40}$. Refiriéndose a este tipo de enseñanzas, A. Ten concluye acertadamente: «Los objetivos, los métodos y sobre todo la extracción social de los alumnos no podían coincidir con los ideales científicos de la Ilustración europea [...] En cuanto a los alumnos ajenos a la comunidad, la conocida política de formación de élites aristocráticas, orientaba sus mejores esfuerzos a la educación de unas capas de la población ideológica, social y económicamente alejadas de cualquier interés real por la aventura científica" ${ }^{41}$. Estas conclusiones también podrían aplicarse a la segunda de las instituciones, el Colegio Imperial. Sin embargo, al menos ésta contiene un elemento novedoso: el hecho de que el gabinete también sirviera para la realización de investigaciones eléctricas, en concreto llevadas a cabo por Christian Rieger, catedrático de matemáticas desde 1761. Los resultados fueron publicados en el opúsculo Observaciones sobre la fuerza eléctrica, grande y fulminante (Madrid, 1763) ${ }^{42}$.

40 Entre 1727 y 1755 pasaron por las aulas del Seminario 314 alumnos, de los cuales solamente 11 permanecieron de 8 a 10 años (la física experimental se ensenaba en el 8. ${ }^{\circ}$; en cambio fueron 128 los que obtuvieron empleos relacionados con su clase, es decir, dentro del ejército, $\mathrm{A}$. LAFUENTE, "La enseñanza de las ciencias durante la primera mitad del siglo XVIII", en VV.AA., Estudios dedicados a Juan Peset Aleixandre, Valencia: Universidad, 1982, vol. II, págs. 489-493.

41 A. E. TEN, " No hay reyno que no sea newtoniano..." Sobre la introducción del newtonismo en Españan, Archives Internationales d'Histoire des Sciences, 131 (1993): pág. 304.

42 Este junto con la obra de Benito Navarro y Abel de Veas, Physica Electrica, o Compendio en que se explican los maravillosos phenómenos de la virtud electrica (s/l, 1752) constituyen los únicos trabajos publicados a partir de investigaciones desarrolladas en España. 
En la segunda etapa destacaron dos instituciones en relación con la física experimental: la Conferencia Físico-matemática-experimental, establecida en Barcelona en $17644^{43}$ y los Reales Estudios de Madrid, fundados en 1770 . La Conferencia no fue concebida propiamente como un centro de enseñanza, sino más bien como una academia dedicada a la promoción e investigación de diferentes ámbitos de la física experimental. Para este cometido se crearon varias "direcciones" (secciones de investigación) que abarcaban los siguientes ámbitos: álgebra y geometría, estática e hidrostática, electricidad y atracciones, óptica, pneumática y acústica, historia natural, botánica, química y agricultura. La principal obligación de los miembros responsables de cada una de las direcciones era la presentación semanal de comunicaciones que informasen de los progresos en cada uno de estos ámbitos científicos. También durante esta primera etapa se dedicaron al estudio de la obra de Musschenbroek. Entre 1782 y 1791, dentro de las ramas relacionadas estrictamente con la física, llegó a tener una cierta actividad la sección de electricidad ${ }^{44}$. Al frente de esta sección se encontraba Antonio Juglá i Font, que había realizado en 1773 una traducción de las Lettres sur l'électricité de Nollet. En el período mencionado presentó cuatro memorias, destacando una de ellas sobre la construcción del pararrayos ${ }^{45}$.

Otro de los aspectos interesantes de este centro fue la promoción de la incipiente industria local de fabricación de instrumentos científicos. En este ámbito destacaron Juan González y José Valls, que fueron nombrados académicos artistas de la Real Academia.

De los diferentes centros de educación superior que contaron con ensefianzas de física experimental, el más importante fue los Reales Estudios ${ }^{46}$.

43 Esta denominación corresponde al período 1764-66; posteriormente, una vez recibida la Real Protección, comenzó a llamarse Real Conferencia Físico-experimental, término que se mantuvo hasta 1770. Finalmente, a partir de ese afio su nombre fue Real Academia de Ciencias Naturales y Artes. Sobre la Conferencia, José Iglesies Fort, "La Real Academia de Ciencias Naturales y Artes", Memorias de la Real Academia de Ciencias y Artes de Barcelona, Barcelona, 1964.

44 Ibid, págs. 35-36.

45 Memoria sobre la construcción y utilidad de los para-rayos (Barcelona, 1787), una de las pocas comunicaciones que llegó a publicarse (Ibid, pág. 35).

46 También algunas universidades consiguieron introducir estas enseñanzas en sus aulas, como la Universidad de Valencia (caso estudiado por A. TEN, "La Física experimental en la universidad espafiola de fines del siglo XVIII y principios del XIX. La Universidad de Valencia y su aula de Mecánica y Física experimental", Llull, 6 (1983): 165-189) y con menor éxito la Universidad de Salamanca (vease G. M. Addy, The Enlightenment in the University of Salamanca, Durham: Duke University Press, 1966, "Plan de Estudios de 1771») y la 
Como ya se ha comentado, esta institución experimentó una profunda reestructuración cuando los miembros de la Companía de Jesús fueron separados de su dirección y docencia (hasta ese momento recibla el nombre de Colegio Imperial). Para llevar a cabo estas reformas se aprobó un nuevo plan de estudios, que incluía, entre otras disciplinas, cátedras de matemáticas, lógica y física experimental; se nombró un tribunal para proveer las cátedras por oposición (que tenían carácter nacional, con igualdad de oportunidades para los concurrentes), y en concreto, para la física experimental, se contrató al mejor fabricante de instrumentos cientificos en esos momentos en Espana: Diego Rostriaga ${ }^{47}$. Como catedrático de esta disciplina (el primero en este país) fue elegido A. Fernández Solano, que ya había impartido la asignatura en el Real Colegio de Cirugía de Cádiz.

Desde su fundación, las enseñanzas impartidas en los Estudios tuvieron un carácter universitario. Su establecimiento obedeció al deseo de las autoridades ilustradas de crear un verdadero centro de formación superior en Madrid que completase los proyectos de reforma pedagógica y científica. Solamente el recelo de otras universidades y el temor a la inevitable competencia impidieron un desarrollo más amplio de estas enseñanzas, recelo que no evitó que en 1787 se reconocieran los cursos seguidos en los RR.EE. El respaldo que recibió desde el gobierno favoreció, entre otras, a la física experimental, considerada como una disciplina emblemática de la modernidad. Este hecho permitió contar con el apoyo económico necesario para la formación del gabinete activo más importante de cuantos existian en esos momentos en España, gabinete que llegó a reunir aproximadamente 240 piezas ${ }^{48}$. En cuanto a los matriculados, después de una primera etapa en la que la asistencia fue muy escasa, a partir de 1787 el número de estudiantes creció significativamente, crecimiento que fue más acusado a partir de los últimos anos del siglo y en la primera década del XIX. En cualquier caso, si lo comparamos con el nivel de asistencia a las clases de matemáticas, la fisica experimental siempre se mantuvo en un lugar discreto.

Universidad de Santiago (véase S. CABEZA DE LEON, Historia de la Universidad de Santiago de Compostela, Santiago, 1947, tomo $2 .^{\circ}$, págs. 96-107).

47 Sobre este fabricante, y en general sobre la fabricación de instrumentos en España, V. GuIjarro, «The Procurement and Manufacture of Scientific Instruments in Spain During the $18^{\text {th }}$ and $19^{\text {th }}$ Centuries", Bulletin of the Scientific Instrument Society, 62 (1999): 7-11.

48 Algunas piezas de este gabinete pueden contemplarse actualmente en el Museo Nacional de Ciencia y Tecnología. 


\section{Conclusión}

Dentro del contexto de la Ilustración, la física experimental desempeńó un papel relevante en el proceso de definición de las ciencias físicas. Después de la restricción de sus contenidos, limitados ahora al mundo inorgánico, y una vez que las investigaciones se centraron en el estudio experimental de los efectos de las propiedades generales y de algunas particulares, la física experimental había conseguido asociarse con las ciencias mecánicas. Así, en correspondencia con la estructura general del conocimiento defendida en esta época, si las observaciones constituyen el contenido de las ideas, el programa general asociado con las ciencias físicas debería comenzar con las disciplinas experimentales (la física) y terminar con las más abstractas (en este caso los saberes matematizados propios de la mecánica). Este programa, sin embargo, no llegaría a difundirse en los centros de educación superior hasta el siglo XIX.

Asimismo la incorporación de esta disciplina en estos centros representó un importante desarrollo de la profesionalización de la figura del físico. En Europa, en 1750 existían, según Desaguliers, 10612 profesores de física experimental ${ }^{49}$. A finales del siglo XVIII, sin embargo, a juzgar por el número de cátedras y cursos que funcionaban en el Continente, esta cifra se había incrementado notablemente.

Por último, la física experimental, por medio de las demostraciones, los cursos y la publicación de manuales, había contribuido a difundir entre el público en general y entre un buen número de artesanos, en particular, el conocimiento de la ciencia y de diversas aplicaciones prácticas relacionadas con ella. Recordemos, por poner un ejemplo, que el mencionado Rostriaga, asistente de Solano en la cátedra de física experimental de los Reales Estudios, construyó, siguiendo las indicaciones de J. Juan, la primera "máquina de fuego" de Newcomen en España ${ }^{50}$. Acerca de este hecho, es preciso indicar, aunque evidentemente no sea esta la única razón que explique este logro, que los manuales de Nollet, Musschenbroek y Desaguliers, con los que Rostriaga

49 Daumas, Les instruments.., opus cit., pág. 184.

so Acerca de los diferentes modelos de máquinas térmicas realizadas en Espana y, en particular, sobre el modelo que aqui se comenta, J. HeLGUERA QUIJADA, "Transferencias de tecnología británica a comienzos de la Revolución Industrial: un balance del caso español a través del sector energéticon, en Juan L. GARCIA HouRCADE et al., Estudios de bistoria de las técnicas, la arqueologia industrial y las ciencias, Valladolid: Junta de Castilla y León, 1998, págs. 89-106. 
estaba familiarizado, contenían secciones dedicadas a explicar diferentes modelos de máquinas movidas por el "fuego". Así pues, también a través de la promoción de la física experimental se consegúa elevar el nivel de conocimientos y preparación de los mecánicos, algo que, teniendo en cuenta que el mundo occidental se encontraba dando los primeros pasos de la Revolución Industrial, se reclamaba cada vez con mayor urgencia desde el ámbito de las manufacturas.

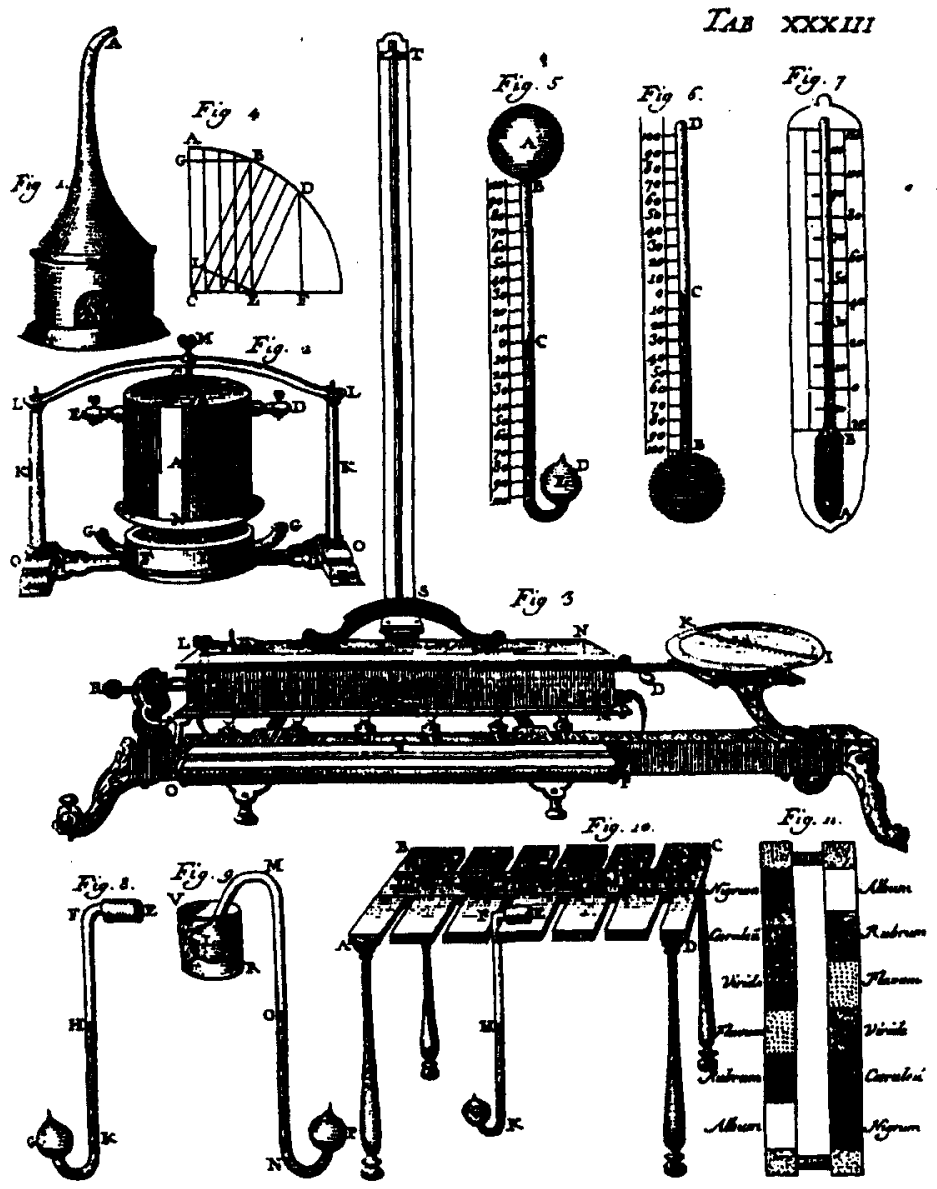

[Fig. 4.] La figura 2 de La ilustración representa una maquina para apreciar la fuerza motriz de vapor de agua en ebullicion, y la figura 3 corresponde a un dilatómetro. Reproducción de la lamina 33. ${ }^{a}$ de la Introductio ad philosophiam naturalem de Musschenbroek. 